\title{
Diagnosis in Networks of Mechatronic Agents: Validation of a Fault Propagation Model and Performance Assessment
}

\author{
Luis Ribeiro, José Barata, Bruno Alves, and João Ferreira \\ Universidade Nova de Lisboa, Faculdade de Ciências e Tecnologia, Campus da FCT-UNL, \\ Monte de Caparica 2829 - 516, Caparica, Portugal \\ \{ldr, jab\}@uninova.pt, bma17241@fct.unl.pt, jpf19013@fct.unl.pt
}

\begin{abstract}
Recent shop floor paradigms and approaches increasingly advocate the use of distributed systems and architectures. Plug-ability, Fault Tolerance, Robustness and Preparedness are characteristics believed to emerge by instantiation of these fundamentally new design approaches. However these features, when effectively present, often come at the cost of a greater system complexity. Enclosed in this complexity increase is a plethora on unforeseen interactions between the entities (modules) that compose the system. The purpose of this paper is, in this context, twofold: to validate a fault propagation model in random networks (that simulate the connectivity of modular shop floor systems) and assess the performance of two diagnostic approaches to expose the impact of relying in local or global information.
\end{abstract}

Keywords: Diagnosis, Complex Systems, Evolvable Systems, Agent-based Manufacturing.

\section{Introduction}

In recent years a considerable effort has been devoted to the research of new paradigms and development of distributed architectures to improve the shop floor response to emerging business requirements and facilitate its integration in the development of suitable and strategic-wise collaborative interactions [1-6].

At the shop floor level the main contributions can be divided in technological and paradigmatic. Industry has been the main driver for the development of platforms that explore emerging Information and Artificial Intelligence technologies (IT/AI) while the Academia has typically provided the conceptual framework that frames the usage of these platforms. Bionic Manufacturing Systems (BMS) [7], Holonic Manufacturing Systems (HMS) [8], Reconfigurable Manufacturing Systems (RMS) [9], Evolvable Assembly Systems (EAS) [10] and Evolvable Production Systems (EPS) [11] are examples of modern control approaches that envision modular systems whose components interact locally and autonomously, within their design purposes and limitations, focusing in attaining a group/social behaviour that exceeds the sum of the individual contributions. Despite the particularities, all the proposals focus in the definition of the modules' interfaces and underlying behaviours that promote a 
self-organized response to production disturbances. In principle, all the main interactions are clearly defined and designed and any unforeseen behaviours are treated as an exception by a fail-safe/escape rule. The variety of systems that can be build from such building blocks is virtually unlimited. While everything seems fit in the design, the heterogeneity of the physical world (the concrete components and their working environment) rather than the paradigmatic abstraction introduce an extra level of complexity in respect to the group dynamics of the given set of components.

Traditional diagnostic tools and approaches have typically been designed to target specific systems and/or conditions. These approaches provide only a limited support for the dynamics envisioned in the paradigms detailed before. In this context, there is a lack of support tools focused both in the analysis of the group dynamics of these systems as well as in diagnosing interactions between the system components from a fault propagation and interference perspectives.

\section{Technological Innovation for Sustainability}

The economical growth generated with the advent of industrialization and mass production kept on feeding an insatiably society demanding low cost reliable goods.

It was believed that mass production/consumption would boost mankind to unprecedented development and sophistication. This would not be verified due to several reasons: technological advances and unscrupulous greed for profit increased unemployment and led to severe social problems; the environmental, health and safety costs were high and the progressive and general increase in customer's welfare made them increasingly demanding in respect to customized goods.

Today's society and business environment are reflexes of such changes. Being competitive is now a matter of organization sustainability. Often perceived as environmental protection, sustainable development goes beyond that and, in a broader sense, is "development that meets the needs of the present without compromising the ability of future generations to meet their own needs" [12]. It is a multi-dimensional and global challenge [13] were business and industry play a relevant role [14].

Mass Customization has been perceived as the excellence paradigm in industry and services it is "the new frontier in business competition for both manufacturing and service industries. At its core is a tremendous increase in variety and customization without a corresponding increase in costs. At its limit is the mass production of individual customized goods and services. At its best, it provides strategic advantage and economic value” [15].

Sustainable development requires a responsible implementation of such paradigm that will certainly include the adoption of innovative organization forms as detailed before. However one has first to master the intricacies of these complex systems in order to regulate them and truly profit from their dynamics maximizing, for the sake of sustainability, fundamental aspects as: equipment re-use (preventing mass disposal), uptime (preventing breakdowns and other sources of energy waste) and efficiency (producing more with less). It is, in this context, unquestionable the role of monitoring and diagnosis in what are anticipated to be the future production systems. 


\section{Related Literature}

Targeting the area of emerging shop floor paradigms the current work gathers contributions from a multitude of areas. From a system architecture perspective the reader is referred to the articles detailed in section 1 and the references therein as well as the following development projects: SIRENA [16] - award winning project that targeted the development of a Service Infrastructure for Real time Embedded Networked Applications [17]. An Implementation of a DPWS stack [18, 19] has been produced under the framework of this project and successfully applied, at device level, in several automation test cases; SODA [20] - creation of a service oriented ecosystem based on the Devices Profile for Web Services (DPWS) framework developed under the SIRENA project; SOCRADES [21] - development of DPWSbased SOA for the next generation of industrial applications and systems; InLife [22] - development of a platform for Integrated Ambient Intelligence and Knowledge Based Services for Optimal Life-Cycle Impact of Complex Manufacturing Assembly Lines and the EUPASS project [23] focused in the study of the application of the multiagent system concept in the domain of micro assembly. The results of the EUPASS project are currently being explored under the FP7 IDEAS project that is focusing in the instantiation of the EPS paradigms in industrial controllers.

From a diagnostic point of view this research positions as complementary to the existing approaches rather than a substitute. As shall be clarified in the forthcoming section, the abstraction level considered for the purpose of performing diagnosis implies the existence of a "first line of diagnosis" that is better supported by conventional approaches. In this context, a complete review on diagnostic methods derived from the automatic control community can be found in [24] where the application of: parameter estimation, evaluation of parity relations, state estimation and principal component analysis methodologies is properly covered. A review of quantitative and qualitative history based methods where diagnosis is performed based on the previous system's faulty behaviour can be found in [25] where the application of artificial neural networks, probabilistic inference methods and expert system is discussed. Qualitative logic based diagnostic methods are covered in [26].

The fault propagation model as well as the framework for network analysis are supported by the study of complex networks $[27,28]$ in particular the proposed model is inspired by the work described in [29] where the conditions for cascading network effects are verified experimentally in random undirected networks.

\section{Performing Diagnosis in Networks of Mechatronic Agents}

When addressing the problem of performing diagnosis in the described systems from the following question arises:

Q1 which methods and tools should be developed to perform diagnosis in highly dynamic systems, like EAS/EPS, that denote physical and logical evolution and adaptation, and ensure the co-evolution/adaption of the diagnostic system without reprogramming or reconfiguring it? 
Two hypothesis were set forth for the purpose of this paper:

H.1 - A self-evolving/adaptable diagnostic system for EAS/EPS can be achieved if intelligent shop floor modules explore local interaction to probabilistically infer and revise their internal states emerging at network level a consistent diagnosis.

The second hypothesis attempts to test the possibility of, rather than using local interaction and the emergent effect, use global knowledge and attempt to explain the fault propagation effect from a global network perspective.

H.2 - A self-evolving/adaptable diagnostic system for EAS/EPS can be achieved if the system composed by intelligent shop floor modules is diagnosed as an whole and the fault propagation is explained globally.

Both systems use as input the connectivity information in the network.

The system for testing hypothesis H.1 has been previously documented in [30-32] where some preliminary informal tests have been carried out to detail the significance of the nature of knowledge representation [30] and the supporting IT infrastructure [31, 32]. The system makes use of a Hidden Markov Model (HMM) [33] that from, a set of 18 possible observations, attempts to infer each agent state.

The system for testing hypothesis H.2 has been previously documented in [34] and uses a temporal logic engine to explain the most probable propagation path. Table 1 compares both approaches.

Table 1. A comparison between the systems under test

\begin{tabular}{|c|c|c|}
\hline Characteristic & H.1 System & H.2 System \\
\hline $\begin{array}{l}\text { The reasoning } \\
\text { process }\end{array}$ & $\begin{array}{l}\text { Each agent attempts to use fault } \\
\text { related local information (direct } \\
\text { neighbors only) to infer its internal } \\
\text { state. The agent can take one of five } \\
\text { possible states that denote whether } \\
\text { the agent is: normal (OK), suffering } \\
\text { an uncorrelated failure (NOK), } \\
\text { propagating a fault from which it is } \\
\text { the origin (PFO), being affected by a } \\
\text { fault generated elsewhere (PFOther) } \\
\text { and a combination of the last two } \\
\text { (PFOPFOther) }\end{array}$ & $\begin{array}{l}\text { All the fault information is } \\
\text { centrally processed. The } \\
\text { diagnostic agent waits for the } \\
\text { fault event to stabilize in the } \\
\text { network and tests the best } \\
\text { combination of system logic } \\
\text { rules that explain the failure. }\end{array}$ \\
\hline $\begin{array}{l}\text { Knowledge } \\
\text { Representation }\end{array}$ & $\begin{array}{l}\text { Each agent processes the information } \\
\text { probabilistically using an HMM that } \\
\text { relates } 18 \text { possible observations with } \\
\text { the five hidden states. The } \\
\text { observations are in the form of the } \\
\text { majority and minority of faulty } \\
\text { neighbors both in inbound and } \\
\text { outbound connections }\end{array}$ & $\begin{array}{l}\text { The information is stored in } \\
\text { logical statements that denote } \\
\text { the influence of a specific } \\
\text { type of component upon } \\
\text { another (e.g conveyor } \\
\text { strongly affects another } \\
\text { conveyor via a mechanic } \\
\text { interaction.) }\end{array}$ \\
\hline
\end{tabular}


Table 1. (Continued)

$\begin{array}{ll}\text { Learning } & \begin{array}{l}\text { Learning is HMM is implementing } \\ \text { support }\end{array} \\ & \text { by } \\ \lambda^{*}=\arg \max _{\lambda} P(O \mid \lambda) .\end{array}$

Learning is necessarily supervised since the a system expert has to validate the training sequences that are used to induce the model.

Complexity The complexity of the diagnostic process is constrained at agent level and independent of the size of the network

Update Each agent performs a new diagnose Dynamics asynchronously when one of its neighbors changes its state. As an whole the system takes some time to stabilize after the fault propagation stabilizes

\section{The Fault Propagation Model}

To assess the limits of both approaches the agents abstracting the shop floor components where ran in fault simulation mode. In simulation mode one agent of the network will initiate a fault that will spread across the system. The propagation rules imply that the fault will always propagate through all possible outbound links departing from an affected agent. In practice this means that the probability that a fault affects distant nodes decays with the distance to the originating node. Some nodes in the network are more likely to be affect by the fault (vulnerable nodes) and are randomly distributed. A vulnerable node has an $80 \%$ chance of being affected as opposed to 5\% chance for the remaining. Being affected by a fault does not necessarily means that it is perceived by the agent's sensor. In this context, sensor fails with a $10 \%$ chance. The percentage of vulnerable nodes was studied from $0 \%$ to
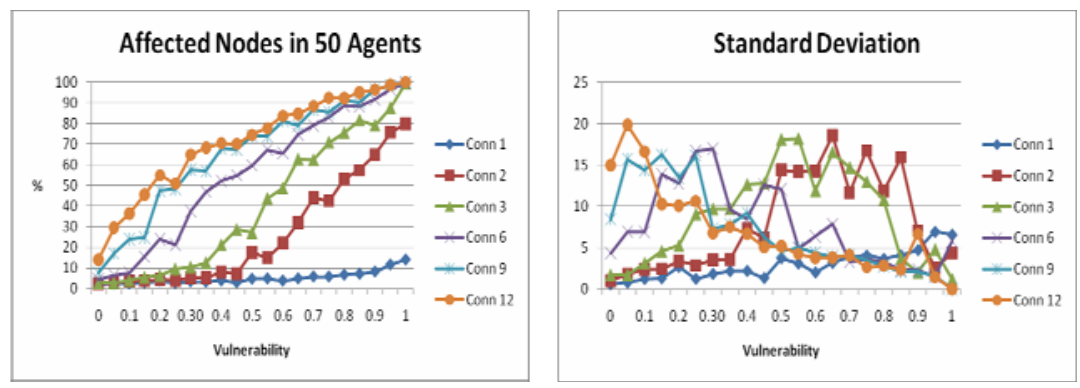

Figs. 1 and 2. The behavior of the propagation model in a network of 50 agents in respect to the percentage of vulnerable nodes (fig. 1). The standard deviation per 100 trials per vulnerability. 
$100 \%$ with $5 \%$ steps for random networks with the following values for average degree: 1, 2, 3, 6, 9, 12. For each value of vulnerability 100 faults (trials) were ran. The number of agents (nodes) considered was 50 .

The results are depicted in Fig. 1 (the average percentage of affected nodes) and Fig. 2 (the standard deviation for each set of 100 trials). The statistical analysis of the results confirms what is intuitively anticipated in systems with such characteristics. The increase in the connectivity causes the response of the network, in respect to the number of affected nodes to shift for approximately exponential (very low values of connectivity), to approximately logarithmic. The standard deviation tends to zero for higher connectivity values as a great majority of the nodes is systematically affected. For low connectivity values the system is more sensible to the distribution of vulnerable nodes and the standard deviation grows in between $40 \%$ and $70 \%$ of vulnerable nodes. This deviation is due to the presence of clusters of vulnerable nodes in some networks.

\section{Comparing Both Approaches}

Both diagnostic approaches were submitted to 300 faults (trials) in networks of 50 agents to assess the performance of both systems in respect to the complexity of the network. When considering the complexity of a network several metrics have been reported in the literature [35]. Given the directed nature of the networks considered a representative measure of complexity is

$\mathrm{C}=\mathrm{A} / \mathrm{V}$ (where $\mathrm{A}$ is the number of links in the network and $\mathrm{V}$ is the number of nodes).

The results of the performance assessment test are resumed in Figs. 3 and 4 where the average value for each set of trials is identified as "H.x System" where $\mathrm{x}$ is the number of the hypothesis under test and the confidence interval is bounded by "Lower Interval H.x" and "Upper Interval H.x" for a degree of confidence of $95 \%$.

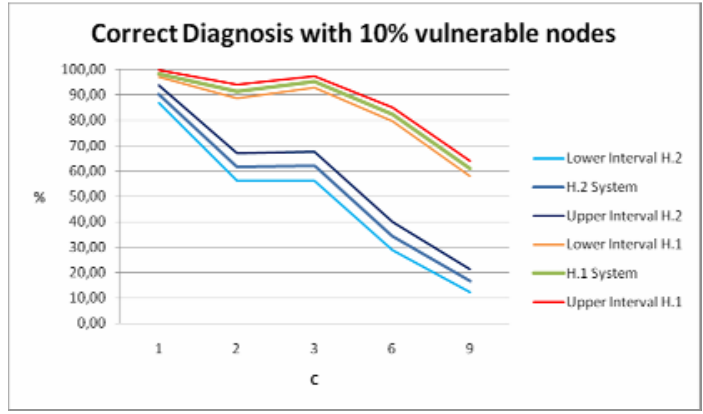

Fig. 3. Results for both systems testing hypothesis H.1 and H.2 with $10 \%$ of vulnerable agents in the network in networks of increasing complexity 


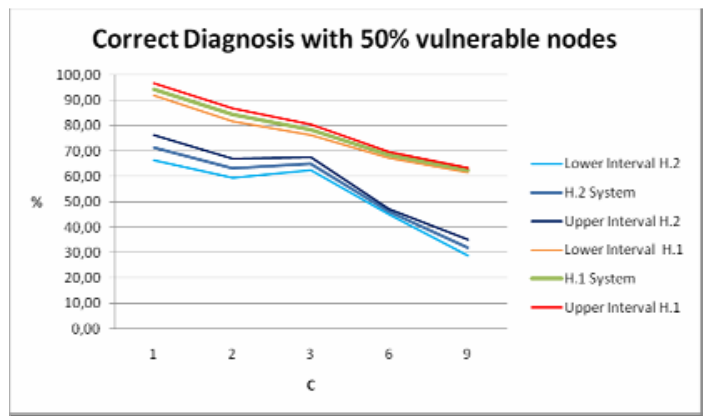

Fig. 4. Results for both systems testing hypothesis H.1 and H.2 with $10 \%$ of vulnerable agents in the network in networks of increasing complexity

The results clearly indicate a degradation of the performance with an increase in the complexity of the system. The performance decrease is more accentuated in the system testing hypothesis H.2. In both cases the performance tends to drop more significantly after $\mathrm{C}=3$. The drop in performance is consistent with the results presented in charts 1 and 2. In fact, beyond complexity or connectivity 3 the network denotes a logarithmic behavior in respect to the number of agents affected by the faults, in both cases under test, yielding an higher number of diagnosis performed. When using local information (H.1) the system essentially fails in the determination of the PFO and PFOther once the higher connectivity promotes the propagation through loops and dramatically shortens the average distance between nodes leading to a fault feedback state where is rather difficult to pinpoint both the origin or the end of the fault. On the global (H.2) case the performance drop can be explained due to the learning and progressive reinforcement of rules. Given the random nature of the faults this system tends to perform better when failures are less pervasive. The H.2 systems learns more meaningful rules in this case. The fact that this system is constantly learning also explains why its performance is better when there are more vulnerable nodes in the network since the rule that all the types of agents affect all the type of agents emerges and stabilizes sooner. The type of fault propagation considered in the tests penalizes, as verified, this second system as it becomes much less probable the existence of fault patterns directly mapped to the logic-based diagnostic rules.

\section{Conclusions and Outlook}

Emerging sustainability challenges are shaping the way in which future shop floors will respond to disturbances and contribute to more rational production patterns. Recent shop floor paradigms have pushed the boundaries of Information Technologies and Artificial Intelligence promoting the integration of components and the seamless reconfiguration of systems. However diagnosis has been left relatively unattended. Current diagnostic approaches are essentially focused in units (either isolated components or entire systems) and some specific parameters. The authors contend that there is added value in considering the interconnectivity of the components as a complementary diagnostic abstraction layer. As the tests expose 
systems can behave differently in respect to their network characteristics and catastrophic failures can emerge even for a reduced number of vulnerable nodes. Understanding how these events may develop in the system and being able to explain them in a network of loosely coupled mechatronic agents is a major challenge that has to be tackled if the emerging shop floor control approaches are to become a reality.

The systems presented and tested attempt to capture this network dimension of the diagnostic problem. The tests range from low network complexity to high, simulating distinct relations between components, typically mechanical/physical interactions in the first case and the communication requirements of networks of mechatronic agents in the second. The generic nature of the tests also raises the question that complexity may not be only present in the emerging paradigms as it may also be perceived in current systems (often perceived as more stable given their supporting technologies). Concerning the two hypothesis under test the system validating hypothesis H.1 ranked higher in the tests denoting more stability in the results and less performance degradation. However, assessing the behavior of diagnostic systems in these complex scenarios (from an interaction perspective) is somehow a novelty and it can be argued that the performance of the system testing hypothesis H.2 could be enhanced by using a distinct technology (arguably so could for the case of H.1). One of the secondary goals of the presented tests was, to a certain extent, perceive whether more conventional approaches (logic based reasoning in the present case) would perform, using off-the-shelve technologies, in diagnosing at the proposed abstraction level. In this matter the results suggest the need for tools and approaches closer to the system implemented to test H.1. Excluding the performance advantage, the use of local information allows embedding the diagnostic system at agent level not corrupting the decoupled nature of the underlying control/configuration logic while promoting scalability (fundamental feature of future production systems).

\section{References}

1. Colombo, A.W.: Industrial Agents: Towards Collaborative Production Automation, Management and Organization. IEEE Industrial Electronics Society Newsletter 52, 17-18 (2005)

2. Camarinha-Matos, L.M.: Collaborative networked organizations: Status and trends in manufacturing. Annual Reviews in Control 33, 199-208 (2009)

3. Camarinha-Matos, L.M., Afsarmanesh, H.: Collaborative networks: a new scientific discipline. Journal of Intelligent Manufacturing 16, 439-452 (2005)

4. Camarinha-Matos, L.M., Afsarmanesh, H.: Elements of a base VE infrastructure. Computers in Industry 51, 139-163 (2003)

5. Camarinha-Matos, L.M., Afsarmanesh, H., Galeano, N., Molina, A.: Collaborative networked organizations - Concepts and practice in manufacturing enterprises. Computers \& Industrial Engineering 57, 46-60 (2009)

6. Stamatis, K., Colombo, A.W., Jammes, F., Strand, M.: Towards Service-oriented Smart Items in Industrial Environments. International Newsletter on Micro-Nano Integration MST News. VDI/VDE-IT, 11-12 (2007)

7. Ueda, K.: A concept for bionic manufacturing systems based on DNA-type information. In: PROLAMAT. IFIP, Tokyo (1992) 
8. Babiceanu, R., Chen, F.: Development and applications of holonic manufacturing systems: a survey. Journal of Intelligent Manufacturing 17, 111-131 (2006)

9. Koren, Y., Heisel, U., Jovane, F., Moriwaki, T., Pritchow, G., Ulsoy, A.G., Van Brussel, H.: Reconfigurable Manufacturing Systems. CIRP Annals - Manufacturing Technology 48, 527-540 (1999)

10. Onori, M.: Evolvable Assembly Systems - A New Paradigm? In: 33rd International Symposium on Robotics Stockholm (2002)

11. Barata, J., Frei, R., Onori, M.: Evolvable Production Systems Context and Implications. In: International Symposium on Industrial Informatics. IEEE, Vigo (2007)

12. WCED: Report of the World Commission on Environment and Development: "Our Common Future" Development and International Economic Co-Operation: Environment. United Nations (1987)

13. Agenda, U.N.: 21. United Nations Conference on Environment and Development. Rio de Janeiro, Brazil (1992)

14. Asif, M., de Bruijn, E.J., Fisscher, O., Steenhuis, H.J.: Achieving sustainability three dimensionally. In: 4th IEEE International Conference on Management of Innovation and Technology, ICMIT 2008, pp. 423-428 (2008)

15. Joseph Pine II, B.: Mass Customization: The New Frontier in Business Competition. Havard Business School Press, Boston (1993)

16. SIRENA: Service Infrastructure for Real-time Embedded Network Applications (2006), http: / / www. sirena-itea.org/Sirena/Home.htm

17. Jammes, F., Smit, H.: Service-oriented architectures for devices - the SIRENA view. In: International Conference on Industrial Informatics, pp. 140-147. IEEE, Perth (2005)

18. Jammes, F., Mensch, A., Smit, H.: Service-Oriented Device Communications Using the Device Profile for Web Services. In: Advanced Information Networking and Applications Workshops, pp. 947-955. IEEE, Los Alamitos (2007)

19. Jammes, F., Mensch, A., Smit, H.: Service-Oriented Device Communications Using the Device Profile for Web Services. In: ACM 3rd International workshop on middleware for pervasive and ad-hoc computing (2005)

20. SODA: Service Oriented Device and Delivery Architecture 2008 (2006 - 2008), http: / /www. soda-itea.org/Home/default.html

21. SOCRADES: Service-Oriented Cross-layer infRAstructure for Distributed smart Embedded devices (2006), http: / / www. socrades.eu/Documents / AllDocuments / default.html

22. InLife: Integrated Ambient Intelligence and Knowledge Based Services for Optimal LifeCycle Impact of Complex Manufacturing and Assembly Lines (2006), http: / / www . uninova.pt/inlife/

23. EUPASS: Evolvable Ultraprecision Assembly Systems (2006), http: / /www. eupass.org/

24. Isermann, R.: Fault Diagnosis Systems: An Introduction from Fault Detection to Fault Tolerance. Springer, Berlin (2006)

25. Venkatasubramanian, V., Rengaswamy, R., Kavuri, S.N.: A review of process fault detection and diagnosis Part III: Process history based methods. Computers and Chemical Engineering 27, 327-346 (2003)

26. Venkatasubramanian, V., Rengaswamy, R., Kavuri, S.N.: A review of process fault detection and diagnosis Part II: Qualitative models and search strategies. Computers and Chemical Engineering 27, 313-326 (2003)

27. Amaral, L.A.N., Ottino, J.M.: Complex networks: Augmenting the framework for the study of complex systems. The European Physical Journal B 38, 147-162 (2004) 
28. Dorogovtsev, S.N., Mendes, J.F.F.: Evolution of networks. Advances in Physics 51, 10791187 (2002)

29. Watts, D.J.: A simple model of global cascades on random networks. Proceedings of the National Academy of Sciences of the United States of America 99, 5766 (2002)

30. Ribeiro, L., Barata, J., Ferreira, J.: The Meaningfulness of Consensus and Context in Diagnosing Evolvable Production Systems. In: Camarinha-Matos, L.M., Pereira, P., Ribeiro, L. (eds.) DoCEIS 2010. IFIP AICT, vol. 314, pp. 143-150. Springer, Heidelberg (2010)

31. Ribeiro, L., Barata, J., Ferreira, J.: Emergent Diagnosis for Evolvable Production Systems. In: IEEE International Symposium on Industrial Electronics, Bari, Italy (2010) (accepted)

32. Ribeiro, L., Barata, J., Ferreira, J.: An Agent-Based Interaction-Oriented Shop Floor to Support Emergent Diagnosis. In: IEEE International Conference on Industrial Informatics. IEEE, Osaka (2010) (accepted)

33. Lawrence, R.R.: A tutorial on hidden Markov models and selected applications in speech recognition. Readings in speech recognition, pp. 267-296. Morgan Kaufmann Publishers Inc., San Francisco (1990)

34. Ribeiro, L., Barata, J., Alves, B.: Exploring the Network Dimension of Diagnosis in Evolvable Production Systems. In: IEEE Emerging Technologies and Factory Automation (ETFA 2010). IEEE, Bilbao (2010) (accepted)

35. Bonchev, D., Buck, G.A.: Quantitative measures of network complexity. Complexity in Chemistry, Biology, and Ecology, 191-235 (2005) 